\title{
Leitthema
}

Monatsschr Kinderheilkd 2014 • 162:137-145 DOI 10.1007/s00112-013-2965-9

Online publiziert: 6. Februar 2014

(c) Springer-Verlag Berlin Heidelberg 2014

Redaktion

G. Hansen, Hannover

M.A. Rose ${ }^{1,2}$

${ }^{1}$ Klinik für Kinder- und Jugendmedizin, Sana-Klinikum Offenbach

${ }^{2}$ Johann Wolfgang Goethe-Universität Frankfurt

\section{Infektionen der oberen Atemwege}

\section{Akute Rhinitis und \\ Rhinosinusitis \\ Akute infektiöse Rhinitis} kungen, aber auch schwerwiegende Verläufe sind möglich. Um diese möglichst zu vermeiden, kommt präventiven Maßnahmen in Form von Impfungen, aber auch dem raschen Erkennen und der adäquaten Behandlung therapiebedürftiger schwerer Infektionskrankheiten der oberen Atemwege entscheidende Bedeutung zu.

Ein gesundes Kind erkrankt altersabhängig durchschnittlich an 5-6 akuten Atemwegsinfektionen pro Jahr, die meist unkompliziert und selbstlimitierend verlaufen und überwiegend durch Viren hervorgerufen werden (• Tab. 1, [19]). Gleichzeitig sind Erkältungen einer der häufigsten Gründe für eine Inanspruchnahme der ambulanten und stationären kinder- und hausärztlichen Versorgung, stellen auch volkswirtschaftlich eine hohe Belastung dar und führen in rund $10 \%$ der Erkrankungsfälle zu verschriebener oder Selbstmedikation, die ihrerseits teilweise von fraglichem Nutzen ist oder sogar zu Folgeschäden führen kann.

Die nachfolgende Übersicht soll zu einem ressourcensparenden und zielführenden Umgang mit akuten Infektionen der oberen Atemwege bei Kindern und Jugendlichen beitragen. Eine Definition der im Folgenden häufig angeführten Evidenzgrade findet sich in $\bullet$ Tab. 2
Sie wird in der Regel durch Viren [u. a. Rhino-, RS- („respiratory syncytial“), Influenza- und Parainfluenza-, Adeno-, Coronaviren] hervorgerufen und ist ein selbstlimitierendes Krankheitsbild, das allerdings bei Säuglingen durch behinderte Nasenatmung zu Trinkschwierigkeiten und Atemstörungen führen kann. Symptomatische Maßnahmen wie nasal verabreichte Muttermilch oder physiologische Kochsalzlösung können Linderung verschaffen (Evidenzgrad C).

Abschwellende Nasentropfen können ebenfalls helfen, sollten aber wegen ihrer Beeinflussung der Schleimhautdurchblutung nur wenige Tage Einsatz finden, und insbesondere bei Säuglingen sind altersgerecht niedrige Konzentrationen zu beachten. Begünstigend für komplizierte Verläufe sind vorbestehende Abwehrschwäche, Fehlbildung und Grunderkrankungen wie Mukoviszidose [zystische Fibrose $(\mathrm{CF})]$.

\section{》) Bei akuter Rhinitis sind meist symptomatische Maßnahmen ausreichend}

Im Zusammenhang mit einer akuten Rhinitis können benachbarte Strukturen mitbetroffen sein und bakteriell superinfizieren (Otitis media, Sinusitis, periorbitale Zellulitis usw.). Ein eitriger Schnupfen stellt keine Indikation für eine Antibiotikatherapie dar. Auch die unkomplizierte Otitis media muss jenseits der ers- ten 2 Lebensjahre bei ansonsten gesunden Kindern nicht primär antibiotisch behandelt werden, sondern spricht häufig gut auf symptomatische Maßnahmen (Ibuprofen, Paracetamol, abschwellende $\mathrm{Na}$ sentropfen) mit einer hohen Selbstheilungsrate an (Evidenzgrad A). Dauert eine akut-infektiöse Rhinitis länger als 10 Tage, sind differenzialdiagnostische Überlegungen ratsam (v. a. Allergien, Fremdkörper).

\section{Entzündungen der \\ Nasennebenhöhlen}

Da die Pneumatisierung der Schädellufträume erst mit zunehmendem Alter erfolgt, präsentieren sich infektiöse Entzündungen der Nasennebenhöhlen je nach Lebensalter unterschiedlich. Kieferhöhlen und Siebbeinzellen sind schon früh angelegt, während Keilbein- und Stirnhöhlen bei den meisten Kindern erst im Vorschul- bzw. Schulalter pneumatisieren und erst dann eine Sinusitis entstehen kann. Typischerweise folgt sie auf eine Erkältung - selten kann sie auch von dentalen Prozessen ausgehen und wird durch Grunderkrankungen (Immundefizienz, ziliäre Dysfunktion, $\mathrm{CF}$ ) begünstigt. Das bakterielle Erregerspektrum umfasst v. a. Pneumokokken, Haemophilus influenzae und Moraxella catarrhalis, seltener A-Streptokokken, bei Abwehrschwachen auch Feuchtkeime (z. B. Pseudomonaden) oder Pilze wie Aspergillen. Der Nachweis von Staphylococcus aureus zeigt eher eine Besiedelung der Nase, denn die Ursache einer Rhinosinusitis an (Evidenzgrad B).

Die Bedeutung von Antibiotika bei der akuten Rhinosinusitis ist umstritten, und Studien ergaben bei unkomplizierten Verläufen unter symptomatischen Maßnah- 
Tab. 1 Virale Erreger der banalen Er-

kältung bei Kindern und Jugendlichen.

\section{(Nach [19])}

\begin{tabular}{|ll}
\hline Virus & Anteil (\%) \\
\hline Rhinovirus & $30-50$ \\
\hline Coronavirus & $10-15$ \\
\hline Influenzavirus & $5-15$ \\
\hline „Respiratory syncytial virus & 5 \\
\hline Parainfluenzavirus & 5 \\
\hline Adenovirus & $<5$ \\
\hline Enterovirus & $<5$ \\
\hline Unbekannt & $20-30$ \\
\hline
\end{tabular}

Tab. 3 Epiglottitis, viraler und bakterieller Krupp: klinische Charakteristika

\begin{tabular}{|lllll}
\hline & & Epiglottitis & Viraler Krupp & Bakterieller Krupp \\
\hline \multirow{2}{*}{ Alter } & Spannbreite & 3 Monate bis 5 Jahre & 6 Monate bis 3 Jahre & 8 Monate bis 14 Jahre \\
\cline { 2 - 5 } & Gipfel & 2,5 Jahre & 1,5 Jahre & 3,8 Jahre \\
\hline \multirow{2}{*}{ Husten } & Kaum & $\begin{array}{l}+++ \\
\text { Bellend, trocken }\end{array}$ & $\begin{array}{l}+++ \\
\text { Produktiv }\end{array}$ \\
\cline { 3 - 5 } & & Spät & Früh & Früh \\
\hline \multirow{2}{*}{ Stridor } & Im Verlauf & Spanehmend & Akut \\
\cline { 2 - 5 } & Beginn & Hochakut & Zunehor & Ja \\
\hline Toxischer Aspekt & Ja & Nein, mildes Fieber & Wenig \\
\hline Dsyphagie & Ausgeprägt & Wenig & Nein \\
\hline Speichelfluss & Ja & Nein & \\
\hline
\end{tabular}

men Spontanheilungsraten bis $\mathrm{zu} 80 \%$ (Evidenzgrad A, B; [4]). Indiziert ist eine Antibiotikatherapie bei Symptompersistenz (Sekretstau, nasale Obstruktion) über 7 bis 10 Tage, schweren Verläufen (Fieber $>39^{\circ} \mathrm{C}$, eitriges Nasensekret, Gesichtsschmerz) an 3 bis 4 aufeinanderfolgenden Tagen oder biphasischem Verlauf mit erneuter Symptomzunahme nach Besserung der Erkältungssymptomatik. Unverändert ist, wie auch bei der Otitis media, Amoxicillin Mittel der Wahl; alternativ können Cefuroximaxetil oder Clarithromycin (cave: Resistenzentwicklung bei zu breiter Anwendung!) angewandt werden. Bei Nichtansprechen kann mit Amoxicillin/Clavulansäure oder Ceftriaxon eskaliert werden ([4], Evidenzgrad A, B).

\section{Infektionen des Oropharynx}

Sie betreffen im Kindesalter in rund 50\% der Fälle den Peritonsillarraum, in rund 20\% den Retropharyngealraum. Bakterielle Tonsillitiden und Peritonsillarabszesse können die oberen Atemwege klinisch relevant verlegen. Typische tonsillitisverursachende Bakterien sind $\beta$-hämolysierende Streptokokken der Gruppe A, Streptococcus pyogenes, Pneumokokken,

Tab. 2 Evidenzgrade für die Belastbarkeit medizinischer Empfehlungen. (Aus [1])
Evidenzgrad A

\begin{tabular}{|ll}
\hline Evidenzgrad B & $\begin{array}{l}\text { Teils unterschiedliche } \\
\text { Studienaussagen } \\
\text { Begrenzt patienten- } \\
\text { orientiert }\end{array}$ \\
\hline Evidenzgrad C & $\begin{array}{l}\text { Expertenkonsens/ } \\
\text {-meinung, übliche } \\
\text { Praxis, Fallserien }\end{array}$ \\
\hline
\end{tabular}

Qualitativ hochwertige patientenorientierte Evidenz Praxis, Fallserien
Staphylococcus aureus sowie selten auch Anaerobier (Fusobacterium spp., Prevoces spp.). Aerobe Bakterien verursachen typischerweise akute Primärinfektionen, wohingegen es bei Anaerobierinfektionen zur Abszessbildung oder Ausbreitung in tiefere und benachbarte Gewebe kommen kann.

Während bei der klassischen Tonsillitis durch aerobe Bakterien unverändert Penizillin angezeigt ist, sollten bei Prozessen, die über die Tonsillen hinausgehen, auch gegen Anaerobier wirksame Antibiotika Verwendung finden (Metronidazol, $\beta$-Laktamase-stabile Antibiotika wie Amoxicillin/Clavulansäure; Evidenzgrad A).

Bei toxischem, schwerkrankem Erscheinungsbild ist darüber hinaus zusätzlich Clindamycin in Erwägung zu ziehen, da hierdurch die Freisetzung bakterieller Exotoxine verhindert werden kann ([26], Evidenzgrad C).

Es gibt unverändert eine Kontroverse, inwieweit bei drohender Verlegung der oberen Atemwege durch entzündete Tonsillen eine frühzeitige chirurgische Intervention hilfreich ist. In jedem Falle sollte bei Abszedierung eine chirurgische Drainage erfolgen, um eine spontane Ruptur tella spp., Porphyromonas spp., Actinomy- und das damit verbundene Aspirationsrisiko zu minimieren, ebenso auch eine Ausbreitung ins Mediastinum oder ins umliegende Gewebe mit Arrosion von Blutgefäßen und lebensgefährlichen Blutungen.

\section{Retro- und Parapharyngealabszesse}

Sie treten typischerweise in Regionen auf, deren Lymphknoten den Nasopharynx, die paranasalen Sinus, das Mittelohr, die Zähne oder benachbarte Knochen drainieren. Retropharyngealabszesse sind insbesondere bei jungen Kindern häufiger, da sich die hierfür relevanten lymphatischen Gewebe mit zunehmender immunologischer Ausreifung zurückbilden und atrophieren. Es besteht eine Wendigkeit zum männlichen Geschlecht (männlich zu weiblich 2:1), das mediane Manifestationsalter liegt bei rund 34 Lebensmonaten.

Die Diagnosestellung erfolgt üblicherweise über Computertomographien (CT; Treffsicherheit 68\%). Typische Leitsymptome sind Fieber, Halsschmerzen, Tortikollis und Nackenschmerz, bei jüngeren Kindern kann auch ein toxisches Erscheinungsbild imponieren. In bis zu 10\% der Fälle entwickelt sich eine Obstruktion der oberen Atemwege. Weitere mögliche klinische Zeichen sind pharyngeale Vorwölbung, Tonsillenverlagerung sowie vermehrter Speichelfluss.

Typische verursachende Erreger sind $\beta$-hämolysierende Gruppe-A-Streptokokken, Staphylococcus aureus (bis zu $30 \%$ methicillinresistent), Moraxella catarrhalis, nicht typisierbare Hämophilusarten, selten auch fakultativ pathogene Keime der oropharyngealen Flora. Durch eine frühzeitige antibiotische Intervention (z. B. Clindamycin) kann eine primäre chirurgische Intervention verhindert werden (Evidenzgrad C).

> Eine Beeinträchtigung der Atemwege wird insbesondere im ersten Lebensjahr beobachtet.

Da in diesen Fällen die endotracheale Intubation durch entzündungsbedingte Veränderungen der Anatomie schwierig sein kann, ist eine Narkosevorbereitung 
unter pädiatrisch-intensivmedizinischen Kautelen sinnvoll (Evidenzgrad B).

Auch bei jungen Kindern sollten geblockte Tuben Anwendung finden, um ein Aspirieren des freitretenden Eiters zu verhindern. Für einen Einsatz von systemischen Steroiden gibt es keine belegte Wirksamkeit. Mehr als 2/3 der Retropharyngealabszesse enthalten $\beta$-Laktamase bildende Organismen, oft auch Anaerobier, was bei der Antibiotikatherapie berücksichtigt werden sollte.

Insbesondere bei jüngeren Patienten ist eine deszendierende Mediastinitis - speziell bei methicillinresistentem Staphylococcus aureus - beschrieben, die eine schwierige Prognose hat (Mortalität 15$50 \%$ ) und eine kalkulierte antibiotische Therapie sowie ein engmaschiges Monitoring des klinischen Verlaufs erfordert.

\section{Seltene, aber wissenswerte Besonderheiten}

\section{Lemierre-Syndrom}

1936 beschrieb Lemierre [17] erstmals diese seltene Komplikation von Pharyngotonsillarinfektionen als „anaerobe postanginale Septicaemie“ in Verbindung mit Infektionen durch Bacillus funduliformis (heutzutage bekannt als Fusobacterium necrophorum) und Bacillus symbiophiles. Typischerweise kommt es infolge der prutriden Pharyngotonsillarinfektion zu einer Thrombophlebitis mit septischer Embolie durch normalerweise saprophytische Anaerobier, wobei die septikämische Phase typischerweise 4 bis 5 Tage nach Beginn der Halsschmerzen mit Fieberanstieg und Nackensteifigkeit einhergeht, während die ursprüngliche pharyngotonsillare Symptomatik schon rückläufig sein kann. Es kann zur Thrombose der ipsilateralen V. jugularis interna kommen, ebenso auch zu einer Streuung in die Lunge, septischer Arthritis und Osteomyelitis, Meningitis, Leber-, Nieren- und Hautabszessen.

Das Lemierre-Syndrom tritt typischerweise bei vorher gesunden Jugendlichen auf, es sind aber auch Fälle bei Säuglingen und Kleinkindern beschrieben. Nachdem die auch als vergessene Krankheit beschriebene Komplikation mit der Verfügbarkeit von Breitspektrumantibiotika kaum noch beobachtet wurde, wird sie mit der zuneh-
Monatsschr Kinderheilkd 2014 · 162:137-145 DOI 10.1007/s00112-013-2965-9

(c) Springer-Verlag Berlin Heidelberg 2014

M.A. Rose

Infektionen der oberen Atemwege

\section{Zusammenfassung}

Hintergrund. Infektionen der oberen Atemwege tragen erheblich insbesondere zur frühkindlichen Morbidität bei, sind die häufigste Ursache für Krankenhausaufenthalte bei unter 4-jährigen Kindern, führen oft zu therapeutischer Polypragmasie und sind eine der häufigsten Verschreibungsursachen für Antibiotika. Ihr Schweregrad variiert von milden, selbstlimitierenden Verläufen bis hin zu potenziell lebensbedrohlichen Atemwegsverlegungen.

Diagnose. Der behandelnde Arzt kann häufig ausschließlich auf der Grundlage von Anamnese und körperlichem Untersuchungsbesatz zusätzlicher diagnostischer Verfahren (Labor, Bildgebung) sollte unklaren Fällen vorbehalten bleiben.

Therapie. Die zu veranlassende Therapie sollte sich an evidenzbasierten Empfehlun-

\section{Upper airway infections}

\section{Abstract}

Background. Upper airway infections contribute significantly to paediatric morbidity and hospitalization especially of young children, are often treated polypragmatically, and are one of the main reasons for antibiotic prescriptions. The severity varies between mild, self-limiting and potentially life-threatening airway obstructions.

Diagnosis. The physician involved can normally make the right diagnosis based on the patient's history and physical findings; additional diagnostic procedures (blood tests, imaging) should be restricted to unclear cases. Therapy. Antibiotic stewardship should be applied. Imminent airway obstruction will refund die richtige Diagnose stellen. Der Ein- gen orientieren. Insbesondere Antibiotika sollten restriktiv eingesetzt werden. Bei drointensivmedizinische Intervention erforderlich. Da sich virale und bakterielle Infektionen unter ähnlichen klinischen Bildern präsentieren können, ist es sinnvoll, die relevanten Krankheitsbilder unter topographischen Gesichtspunkten abzuhandeln.

Prävention. Durch konsequente Umsetzung der STIKO-Empfehlungen (STIKO: Ständige Impfkommission am Robert Koch-Institut) lässt sich ein erheblicher Teil schwerer Atemwegsinfektionen verhindern.

Schlüsselwörter

Obere Atemwegsinfektionen - Rhinosinusitis . Lemierre-Syndrom · Ludwig-Angina .

Selbstmedikation hender Verlegung der Atemwege wird eine menden Zahl chronisch Kranker und Abwehrschwacher in den letzten Jahren wieder vermehrt beschrieben. Bei unklaren septischen Krankheitsbildern sollte daher immer eine Inspektion des äußeren sowie des inneren Halses erfolgen.

Die Behandlung umfasst i.v. hochdosiertes Penizillin mit Metronidazol oder Clindamycin allein, jeweils über 2 bis 6 Wochen, sowie ggf. Heparinisierung (Evidenzgrad C). quire early and competent paediatric intensive care interventions. Since viral and bacterial upper airway infections can present with similar features, it is useful to approach them under topographical aspects.

Prevention. Following immunization recommendations can prevent an enormous amount of severe potentially life threatening airway infections.

\section{Keywords}

Upper airway infections $\cdot$ Rhinosinusitis . Lemierre's syndrome - Ludwig's angina . Over-the-counter drugs

\section{Ludwig-Angina (Angina gangraenosa)}

Es handelt sich um eine diffuse Infektion des Submandibular- und Sublingualraums mit reduziertem Allgemeinzustand, starken Schmerzen, Fieber und Dysphagie bis hin zur Atemwegsobstruktion. Sie steht typischerweise mit Zahnkaries, Sichelzellkrankheit, Abwehrschwäche und Verletzungen im Kopf-Hals-Bereich in Verbindung und wird durch Staphylokokken, $\alpha$ hämolysierende Streptokokken und Anaerobier (Bacteroides, Peptokokken, Peptostreptokokken) hervorgerufen. 


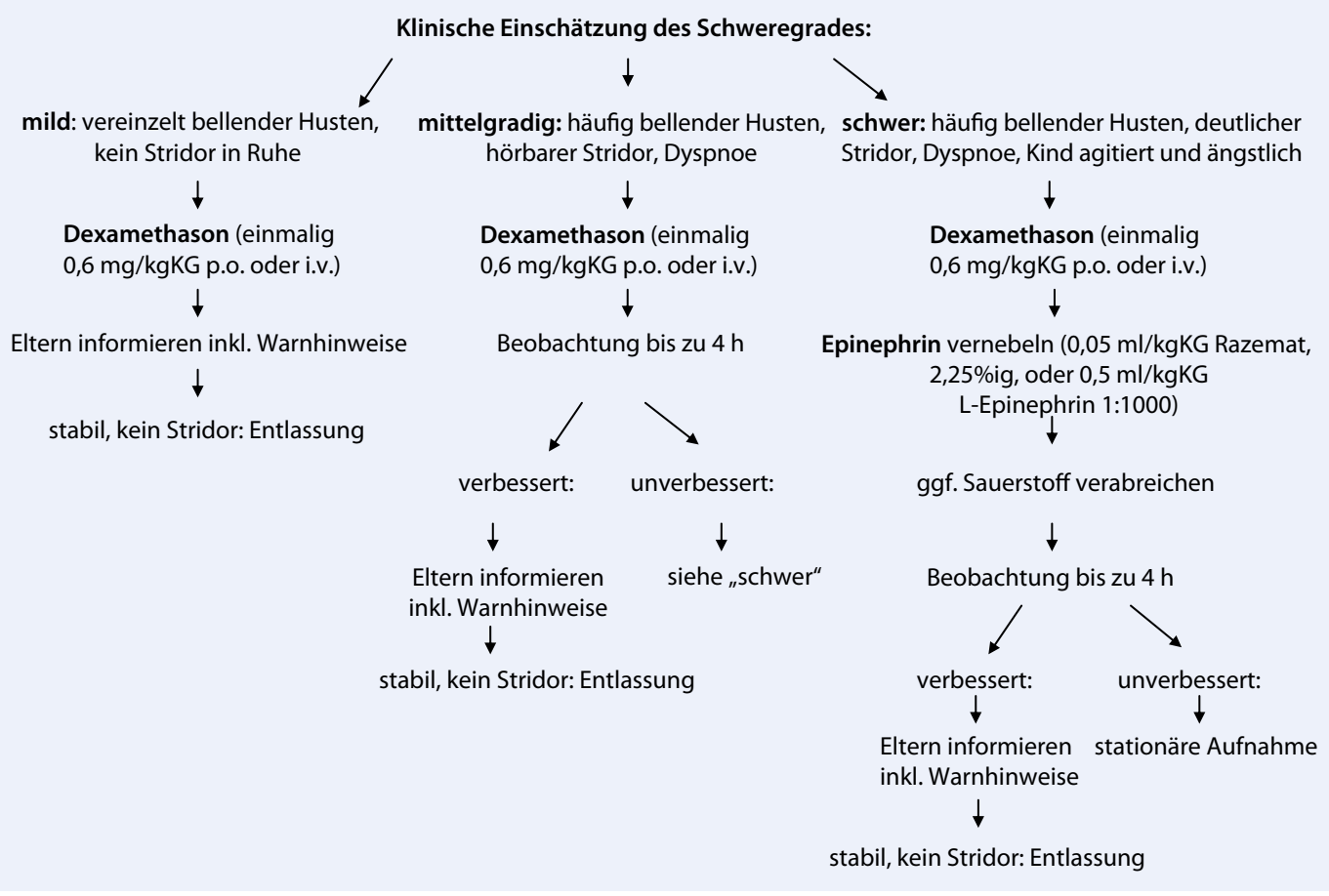

Abb. 14 Ambulante Versorgung von Kindern mit Krupp-Syndrom, KG Körpergewicht. (Nach [30])
Die antibiotische Therapie kann mit Metronidazol und/oder $\beta$-Laktamase -stabilen $\beta$-Laktam-Antibiotika erfolgen, bei Penizillinunverträglichkeit mit Clindamycin. Bei ausgeprägten Gewebsschwellungen können Glukokortikoide erwogen werden, bei entsprechenden Eiteransammlungen die chirurgische Drainage (Evidenzgrad B).

\section{Larynx}

\section{Larynxpapillomatose}

Die rekurrierende Larynxpapillomatose wird durch das humane Papillomavirus (HPV, typischerweise Typen 1 und 11) hervorgerufen, welches auch $90 \%$ der Genitalwarzen verursacht. Rund 25\% aller Frauen im gebärfähigen Alter tragen HPV in ihrem Genitaltrakt, und eine InUtero-Übertragung ist beschrieben, sodass durch einen Kaiserschnitt allenfalls eine Transmissionsverminderung erreicht werden kann. Die seltene Larynxpapillomatose hat eine Prävalenz von 3-5 auf 100.000 und kann sich bis in die tiefen Atemwege ausdehnen.

Typischerweise beginnt die klinische Präsentation mit Heiserkeit und schreitet langsam voran, bei HPV-Typ 11 sind deutlich fulminantere Verläufe beschrieben.
An Therapieoptionen sind Laserablationen oder endoskopische Mikrodébridements beschrieben, adjuvant Interferon, Ribavirin und Aciclovir (Evidenzgrad C). In den letzten Jahren wird in therapierefraktären Fällen auch zunehmend intraläsional Cidofovir instilliert, bei intrapulmonalem Befall auch systemisch (Evidenzgrad C). Im Tierversuch wurde eine möglicherweise erhöhte Karzinogenese gezeigt, die Übertragbarkeit dieses Risikos auf den Menschen ist unklar [28].

Mit der Verfügbarkeit der quadrivalenten HPV-Impfung gegen die Serotypen $6,11,16$ und 18 bleibt zu hoffen, dass die Krankheit zunehmend zurückgedrängt werden wird.

\section{Epiglottitis}

Mit der Schutzimpfung gegen Haemophilus influenzae Typ B wurde der Haupterreger der akuten Epiglottitis weitgehend ausgerottet. Vereinzelt noch beobachtete Fälle werden durch Haemophilus parainfluenzae, Gruppe-A-Streptokokken, Pneumokokken oder Staphylokokken, bei immunsupprimierten $\mathrm{Pa}$ tienten auch durch Candida spp., Herpessimplex-Virus Typ 1, Varicella-zoster- und Parainfluenzavirus verursacht.

\section{1) Bei entsprechenden \\ Hinweisen ist eine akute Epiglottitis in Betracht zu ziehen}

Bei hoch fiebernden schwerkranken Kindern mit Dysphagie, vermehrtem Speichelfluss, Ängstlichkeit und typischer Sitzposition (extendierter Kopf mit vorgebeugtem Sitz und Abstützen mit den Armen) ist auch heute noch eine akute Epiglottitis zu berücksichtigen ( $\bullet$ Tab. 3). In diesem Fall sollte eine inhalative Narkoseeinleitung unter Notfalltracheotomiebereitschaft und Gabe von Breitspektrumantibiotika erfolgen (Evidenzgrad B).

In unklaren Fällen kann eine seitliche Röntgenaufnahme des Halses (sog. Daumenzeichen) hilfreich sein, keinesfalls sollte aber eine Bildgebung die Sicherstellung des Atemwegs verzögern; die Diagnose muss im Zweifelsfall klinisch gestellt werden.

Bei der Wahl eines Antibiotikums sind lokale Resistenzen zu berücksichtigen; ein Drittgenerationszephalosporin oder ein $\beta$-Laktamase-stabiles Penizillin sind Mittel der Wahl. Über die Rolle systemischer Steroide gibt es eine fortbestehende Kontroverse - nach Studienlage haben sie allerdings keinen Einfluss auf die Notwendigkeit und die Dauer einer Intubation, 


\begin{tabular}{|c|c|c|c|c|}
\hline Krankheitsbild & Anamnese & Untersuchung & Vorgehen & Ätiologie \\
\hline $\begin{array}{l}\text { Virales Krupp- } \\
\text { Syndrom }\end{array}$ & $\begin{array}{l}\text { Bellender Hus- } \\
\text { ten, Schnupfen }\end{array}$ & $\begin{array}{l}\text { Leichtes Fieber, } \\
\text { inspiratorischer } \\
\text { Stridor, Einzie- } \\
\text { hungen, Nasen- } \\
\text { flügeln }\end{array}$ & $\begin{array}{l}\text { Ruhe bewahren } \\
\text { Steroide, ggf. Epine- } \\
\text { phrin verabreichen }\end{array}$ & $\begin{array}{l}\text { Influenza- und } \\
\text { Parainfluenza- } \\
\text { viren der Typen } \\
1-3, \text { RSV }\end{array}$ \\
\hline Epiglottitis & $\begin{array}{l}\text { Halsschmerzen, } \\
\text { rascher An- } \\
\text { fang, kloßige } \\
\text { Sprache, } \\
\text { Speichelfluss, } \\
\text { unvollständiger } \\
\text { Impfstatus?? }\end{array}$ & $\begin{array}{l}\text { Hohes Fieber, } \\
\text { toxischer } \\
\text { Aspekt, Kind } \\
\text { in Atemhilfs- } \\
\text { position }\end{array}$ & $\begin{array}{l}\text { Ggf. seitliche Rönt- } \\
\text { genaufnahme des } \\
\text { Halses, Entzündungs- } \\
\text { parameterbestim- } \\
\text { mung, bei hoch- } \\
\text { gradigem Verdacht } \\
\text { unnötige Aufregung } \\
\text { vermeiden, keine Ra- } \\
\text { cheninspektion }\end{array}$ & $\begin{array}{l}\text { Haemophilus } \\
\text { influenzae Typ B, } \\
\text { hämolysierende } \\
\text { Gruppe-A-Strep- } \\
\text { tokokken }\end{array}$ \\
\hline $\begin{array}{l}\text { Bakterielle La- } \\
\text { ryngotracheitis }\end{array}$ & $\begin{array}{l}\text { Beschwerden } \\
\text { zunehmend, } \\
\text { dann binnen } 3 \\
\text { bis } 5 \text { Tagen de- } \\
\text { kompensierend }\end{array}$ & $\begin{array}{l}\text { Hohes Fieber, } \\
\text { toxischer } \\
\text { Aspekt, produk- } \\
\text { tiver Husten, } \\
\text { viel Sekret, Ein- } \\
\text { ziehungen }\end{array}$ & $\begin{array}{l}\text { Entzündungspara- } \\
\text { meterbestimmung, } \\
\text { Erregernachweis aus } \\
\text { Sekreten, ggf. seitliche } \\
\text { Röntgenaufnahme } \\
\text { des Hals }\end{array}$ & $\begin{array}{l}\text { Staphylococcus } \\
\text { aureus, Haemo- } \\
\text { philus influenzae, } \\
\text { Gruppe-A-Strep- } \\
\text { tokokken }\end{array}$ \\
\hline $\begin{array}{l}\text { Fremdkörper- } \\
\text { aspiration }\end{array}$ & $\begin{array}{l}\text { Plötzlicher Hus- } \\
\text { ten nach sog. } \\
\text { Verschlucken }\end{array}$ & $\begin{array}{l}\text { Stridor, seiten- } \\
\text { differentes } \\
\text { Atemgeräusch }\end{array}$ & Bronchoskopie & Fremdkörper \\
\hline Angioödem & $\begin{array}{l}\text { Paroxysmale } \\
\text { Schwellung, } \\
\text { evtl. Bauchweh }\end{array}$ & $\begin{array}{l}\text { Schwellung von } \\
\text { Gesicht/Nacken }\end{array}$ & $\begin{array}{l}\text { Allergologische und } \\
\text { C1-Esterase-Inhibitor- } \\
\text { Abklärung }\end{array}$ & $\begin{array}{l}\text { Allergische Reak- } \\
\text { tion, C1-Esterase- } \\
\text { Inhibitor-Mangel }\end{array}$ \\
\hline $\begin{array}{l}\text { Peritonsillar- } \\
\text { abszess }\end{array}$ & $\begin{array}{l}\text { Schluckstörung, } \\
\text { Halsweh oft } \\
\text { einseitig }\end{array}$ & $\begin{array}{l}\text { Tonsillen- und } \\
\text { Uvulaverlage- } \\
\text { rung, Enan- } \\
\text { them, Exsudate }\end{array}$ & $\begin{array}{l}\text { Sonographie, ggf. CT } \\
\text { mit Kontrastmittel }\end{array}$ & $\begin{array}{l}\text { Gramnegative } \\
\text { und -positive Or- } \\
\text { ganismen, cave: } \\
\beta \text {-Laktamase- } \\
\text { Bildner, Anae- } \\
\text { robier }\end{array}$ \\
\hline $\begin{array}{l}\text { Retropharyn- } \\
\text { gealabszess }\end{array}$ & $\begin{array}{l}\text { Fieber, Schluck- } \\
\text { störung und } \\
\text {-schmerz, Hals- } \\
\text { weh }\end{array}$ & $\begin{array}{l}\text { Speichelfluss, } \\
\text { Stridor, Nacken- } \\
\text { schwellung, } \\
\text { Pseudomenin- } \\
\text { gismus }\end{array}$ & $\begin{array}{l}\text { CT (lateraler Hals), ggf. } \\
\text { Kontrastmittel }\end{array}$ & $\begin{array}{l}\text { Gramnegative } \\
\text { und -positive Or- } \\
\text { ganismen, cave: } \\
\beta \text {-Laktamase- } \\
\text { Bildner, Anae- } \\
\text { robier }\end{array}$ \\
\hline $\begin{array}{l}\text { Spasmodischer } \\
\text { Krupp }\end{array}$ & $\begin{array}{l}\text { Häufig rezidi- } \\
\text { vierende kurze } \\
\text { Episoden bel- } \\
\text { lenden Hustens }\end{array}$ & $\begin{array}{l}\text { Afebril, milde } \\
\text { Dyspnoe- } \\
\text { zeichen }\end{array}$ & $\begin{array}{l}\text { In unklaren Fällen } \\
\text { endoskopische Ab- } \\
\text { klärung }\end{array}$ & $\begin{array}{l}\text { Siehe viraler } \\
\text { Krupp } \\
\text { Differenzialdia- } \\
\text { gnosen: Allergie, } \\
\text { GÖR }\end{array}$ \\
\hline \multicolumn{5}{|c|}{ CT Computertomographie, GÖR gastroösophagealer Reflux, RSV „respiratory syncytial virus“ } \\
\hline
\end{tabular}

eine intensivmedizinische oder Krankenhausbehandlung [11]. In klinischen Studien [11] ergaben sich sogar Hinweise auf einen verlängerten Krankenhausaufenthalt bei Steroidanwendung, was allerdings dahingehend Ausdruck eines Selektionsbias sein kann, dass schwerer kranke Patienten Steroide erhielten.

\section{Trachea}

Krupp

\section{Allergisches und virales Krupp-Syndrom}

Das praktische Vorgehen bei der ambulanten Versorgung von Kindern mit Krupp-Syndrom geht aus • Abb. 1 hervor.

Neben dem im Sommer insbesondere bei Kindern mit atopischer Disposition auftretenden allergischen Krupp, der typischerweise nach Pollenflug beobachtet wird und sich mit einer eher milden Symptomatik und Rhinoconjunctivitis allergica darstellt, sind virale und bakterielle stenosierende Laryngotracheitiden beschrieben (- Tab. 4). Der allergische Krupp ist in der Regel ein kurzzeitiges selbstlimitierendes Krankheitsbild, das gut auf Antihistaminika anspricht.

$\nabla$

Der virale Krupp kommt in der kinderärztlichen Praxis häufig vor.

Er ist charakterisiert durch den typischen bellenden Husten, Heiserkeit und zunehmenden inspiratorischen Stridor. Im Verlauf können sub- und interkostale Einziehungen und auch exspiratorischer Stridor auftreten. Typischerweise erkranken 6 Monate bis 3 Jahre alte Kinder (Manifestationsgipfel im zweiten Lebensjahr), ursächlich werden insbesondere Parainfluenzaviren, RSV (v. a. bei Säuglingen), Influenzavirus A und B sowie selten $M y$ coplasma pneumoniae (v. a. bei Vorschulkindern) isoliert. Mit neueren Methoden wurde auch humanes Metapneumo-, Corona- und Bocavirus nachgewiesen (• Tab.5).

Das Krankheitsbild ist in der Regel von benignem Verlauf, die Kinder können fiebern, bieten aber insgesamt einen nicht erheblich beeinträchtigten Aspekt. Labormedizinische und radiologische Untersuchungen sind in der Regel entbehrlich, vielmehr sollten die Kinder in einer ruhigen und möglichst ungestörten Atmosphäre betreut werden. Während sich die traditionell eingesetzte Luftbefeuchtung in Metaanalysen nicht als relevant hinsichtlich des klinischen Ausgangs erwies [30], finden unverändert systemische Steroide sowie vernebeltes Epinephrin eine Anwendung (Evidenzgrad A, • Tab. 6). Systemische Steroide führten in klinischen Studien zu einer signifikanten Reduzierung der Notwendigkeit einer Intubation und künstlichen Beatmung [13].

Glukokortikoide. Sie können vernebelt werden (Budesonid $2 \mathrm{mg}$ ), rektal, intramuskulär oder bei Vorhandensein einer Verweilkanüle auch i.v. verabreicht werden. Häufig Anwendung findet Prednisolon [1 mg/kgKG (KG: Körpergewicht)], 
Tab. 5 Klinische Präsentation des viralen Krupp-Syndroms nach Erregern. (Nach [30])

\begin{tabular}{llll}
\hline Ätiologie & Vorkommen & Schwere & Inzidenz Gipfel \\
\hline $\begin{array}{l}\text { Parainfluenzavirus } \\
\text { Typen 1-3 }\end{array}$ & Häufig & $\begin{array}{l}\text { Variabel (schwer bei } \\
\text { Typ 3) }\end{array}$ & Winter, Frühjahr \\
\hline Enterovirus & Manchmal & Meist mild & Herbst \\
\hline Humanes Bocavirus & Manchmal & Meist mild & Frühjahr, Herbst \\
\hline $\begin{array}{l}\text { Influenzavirus A } \\
\text { und B }\end{array}$ & Manchmal & Variabel (schwer bei A) & Winter \\
\hline RSV & Manchmal & Mild bis moderat & Winter \\
\hline Rhinovirus & Manchmal & Meist mild & Frühjahr \\
\hline Adenovirus & Selten & Mild bis moderat & Winter \\
\hline Masernvirus & Sehr selten & Moderat bis schwer & Bei Ausbrüchen \\
\hline RSV „respiratory syncytial virus & & \\
\hline
\end{tabular}

Tab. 6 Evidenzbasierte klinische Emp-

fehlungen beim Krupp-Syndrom. (Nach

[30])

\begin{tabular}{|ll}
\hline $\begin{array}{l}\text { Evidenzbasierte klinische } \\
\text { Empfehlungen }\end{array}$ & $\begin{array}{l}\text { Evidenz- } \\
\text { grad }\end{array}$ \\
\hline $\begin{array}{l}\text { Feuchtluftinhalation beeinflusst } \\
\text { nicht Symptome bei mildem bis } \\
\text { moderatem Krupp-Syndrom im } \\
\text { ambulanten Umfeld. }\end{array}$ \\
\hline $\begin{array}{l}\text { Kortikosteroide helfen beim } \\
\text { Krupp-Syndrom, auch in leichten } \\
\text { Fällen. }\end{array}$ \\
\hline $\begin{array}{l}\text { Die einmalige Gabe oraler Steroi- } \\
\text { de ist bei Patienten mit mildem } \\
\text { Krupp-Syndrom wirksam. }\end{array}$ \\
\hline $\begin{array}{l}\text { Vernebeltes Epinephrin verbes- } \\
\text { sert den Verlauf bei Patienten } \\
\text { mit mittelschwerem bis schwe- } \\
\text { rem Krupp. }\end{array}$ \\
\hline
\end{tabular}

für Dexamethason $(0,15-0,6 \mathrm{mg} / \mathrm{kgKG})$ spricht seine lange Halbwertszeit (antientzündliche Wirkung bis zu $72 \mathrm{~h}$ ). Eine einmalige Verabreichung scheint hinreichend zu sein ([7], • Tab.6).

Epinephrin. Vernebeltes Epinephrin hat eine Wirkdauer von bis zu $2 \mathrm{~h}$ und verkürzte in Studien signifikant die Krankenhausbehandlung (Evidenzgrad A; - Tab. 6, [30]). Razemisches Epinephrin $2,25 \%(0,05 \mathrm{ml} / \mathrm{kgKG})$ scheint genauso wirksam zu sein wie das üblicherweise verfügbare L-Epinephrin (1:1000 verdünnt, 0,5 ml/kgKG), allerdings hält die Wirkung von L-Epinephrin tendenziell länger an, und eine systematische Übersichtsarbeit kam zu dem Ergebnis, dass $3-5 \mathrm{ml}$ reines 1:1000 verdünntes L-Epinephrin sicher und wirksam sind $[2,29]$.

Obwohl die Adrenalininhalation in systematischen Studien [2] ebenfalls als sicher beurteilt wurde, gibt es klinische Be- obachtungen, dass die therapeutisch wirksame Vasokonstriktion über einen nachfolgenden Rebound zu einer Verschlechterung der Atemwegssituation mit kritischer Atemwegsverlegung führen kann. Daher sollte Adrenalin bei instabilen Kindern nicht im ambulanten Kontext inhaliert werden. Im Zweifelsfall kann auch bei schweren Verläufen eines vermutlich viralen Krupps eine zusätzliche Antibiotikatherapie in Erwägung gezogen werden.

Weitere Maßnahmen. Bei ausbleibender Besserung ist eine Verabreichung von Helium-Sauerstoff-Gemisch (Heliox) zu erwägen (Evidenzgrad C). Sollte eine endotracheale Intubation nötig sein, ist diese unter maximalen Sicherheitskautelen und HNO-Tracheotomiebereitschaft durchzuführen. Zur Einleitung sind nichtirritierende Inhalationsnarkotika wie Sevofluran oder Halothan zu bevorzugen. Endotrachealtuben sollten eine Größe unter der altersbezogen erwarteten Größe bereitgehalten werden. Ein im Verlauf zunehmendes Beatmungsleck zeigt einen Rückgang der subglottischen Schwellung.

Sollte der klinische Verlauf über $48 \mathrm{~h}$ keine deutliche Stabilisierung zeigen, sind weitere Steroidgaben zu erwägen, üblicherweise kommt es zur Ausheilung binnen 1 Woche.

Differenzialdiagnose. Differenzialdiagnostisch sind Fremdkörperaspirationen, Epiglottitis, bakterielle Tracheitis, Tonsillitis und Peritonsillar- sowie Retropharyngealabszesse und bei Erkältungen dekompensierende Tracheobronchomalazie oder vaskuläre Kompressionssyndrome zu berücksichtigen.
Prävention. Präventivmedizinisch findet die gut wirksame, ab dem vollendeten 2. Lebensjahr zugelassene nasale mukosale Grippeimpfung (LAIV) Einsatz. Klinische Beobachtungen aus Ländern mit einem breiten Einsatz derselben zeigen im Rahmen der Verhinderung akuter viraler Atemwegsinfektionen benefitäre Nebeneffekte [8].

\section{Bakterielle Laryngotracheitis (bakterieller Krupp)}

Diese wichtige Differenzialdiagnose zur akuten Epiglottitis und viralen Krupperkrankung rückte in den letzten Jahren zunehmend ins Bewusstsein. Eine über 10 Jahre durchgeführte US-amerikanische Untersuchung untermauert die große Bedeutung der bakteriellen Tracheitis für klinisch schwere Infektionen der oberen Atemwege bei Kindern [11].

\section{》) Typisch für bakteriellen Krupp sind Husten, Stridor, Pyrexie und toxischer Aspekt}

Durch die erfolgreichen Schutzimpfprogramme gegen Haemophilus influenzae Typ B und die Behandlungsoption von Glukokortikoiden beim viralen Krupp kam es zu einer relativen Zunahme der bakteriellen Tracheitiden bei den pädiatrisch-intensivmedizinischen Patienten. Häufig geht diesen eine Erkältungskrankheit voran, Kinder mit bakteriellem Krupp imponieren allerdings durch Husten, Stridor, Pyrexie und toxischen Aspekt. Im Fall einer endoskopischen Inspektion finden sich ausgeprägte Erytheme, Ödeme und zähe purulente Sekrete, die an Pseudomembranen erinnern können.

Mikrobiologisch steht Staphylococcus aureus im Vordergrund, aber auch Streptococcus pneumoniae, Gruppe-A-Streptokokken, nichtbekapselte Haemophilus spp. und Moraxella catarrhalis kommen als Erreger in Frage. Interessanterweise geht die durch Moraxella catarrhalis bedingte Laryngotracheitis mit einer erhöhten Intubationsrate einher [21] und wird häufig in Gesellschaft mit Influenza A oder B beobachtet, was ebenfalls die Bedeutung einer Immunisierung auch gesunder Kinder gegen Influenzaviren untermauert. 
Der bakterielle Krupp wird antibiotisch mit einem staphylokokkenwirksamen Drittgenerationszephalosporin behandelt. Glukokortikoide haben keine nachgewiesene Wirksamkeit, vielmehr können sie bei der Entwicklung einer Sepsis mit Pneumonie und Atemnotsyndrom aggravieren. Bei respiratorischer Verschlechterung ist eine endotracheale Intubation unter fiberoptischer Endoskopie und HNO-Tracheotomiebereitschaft angezeigt. In diesem Rahmen kann auch eine Bronchialtoilette erfolgen, die seltenen Fälle von exsudativen Pseudomembranen (exsudative Tracheitis) können ein bronchoskopisches Débridement erfordern (cave Perforation).

\section{Diphtherie (diphtherischer Krupp)}

Ungeachtet der Erfolge laufender Impfprogramme bleibt Diphtherie weltweit ein Problem. Die durch Corynebacterium diphtheria und Corynebacterium ulcerans hervorgerufene Erkrankung beginnt üblicherweise mit kruppösen Beschwerden, Husten und Halsschmerzen mit Voranschreiten zu einem septischen Krankheitsbild mit disseminierter intravasaler Koagulation und Nierenversagen, Erstickungsanfällen durch die Pseudomembranen im oberen Atemwegstrakt, Exotoxinausschüttung mit Lähmungserscheinungen, kardialer Beeinträchtigung mit Erregungsleitungsstörungen und Myokardversagen, Nebennierenrindeninsuffizienz mit endokrinologischem Dekompensieren bis hin zum Versterben. Schon in der ersten Erkrankungswoche können Elektrokardiogrammauffälligkeiten imponieren, die Inzidenz von begleitenden Kardiomyopathien beträgt bis zu 20\% mit einer Mortalität von bis zu 50\% [15].

> Auch heute noch ist die Diphtherie selbst unter adäquater Behandlung mit einer Mortalität von bis zu $10 \%$ vergesellschaftet.

Das Vorhandensein von Pseudomembranen und der sog. Cäsarenhals sind klinische Vorhersagezeichen für die Entwicklung einer Kardiomyopathie [15]. Die Behandlung umfasst unverändert die Gabe von spezifischem Antitoxin, hochdosiertem Penizillin oder Makrolidantibiotika und i.v. Hydrokortison bei ausgepräg- tem Halsödem. Bei kritischer Obstruktion der Atemwege durch Pseudomembranen ist eine Tracheotomie in Erwägung zu ziehen.

Auch wenn in Europa die Diphtherie durch Immunisierungsprogramme weitgehend unter Kontrolle ist, werden immer wieder eingeschleppte Fälle aus nichtindustrialisierten Ländern beobachtet, sodass das Krankheitsbild unverändert bekannt sein muss und auch eine konsequente Immunisierung der in unseren Breitengraden lebenden Bevölkerung weiterhin angezeigt ist.

\section{Erkältungskrank- heiten bei Kindern}

Erkältungen zählen zu den häufigsten Infektionskrankheiten überhaupt; Kinder in den ersten 5 Lebensjahren erleiden 6 bis 8 Episoden/Jahr mit einer Wendigkeit zum männlichen Geschlecht, während Jugendliche und Erwachsene 2- bis 4-mal pro Jahr erkranken. Der Besuch von Gemeinschaftseinrichtungen erhöht das Risiko, wovon auch jüngere Geschwisterkinder daheim (Säuglinge!) betroffen sind.

\section{Erreger und Pathogenese}

Ursächlich sind zwar rund 200 Viren mit je nach Patientenalter unterschiedlicher Verteilung, aber selbst in Studien gelingt in bis zu 30\% der Fälle kein Erregernachweis [9]. Eine in Finnland an akut erkälteten, durchschnittlich 2-jährigen, in Kindertagesstätten betreuten Kindern durchgeführte Studie zeigte Rhinovirus als häufigsten Erreger (71\%), gefolgt von humanen Bocaviren (HBoV; 14\%), Adenovirus (12\%), Enterovirus (10\%), Coronavirus (6\%), Influenzaviren (6\%), RSV (4\%), Parainfluenzavirus $(4 \%)$ und humanes $\mathrm{Me}$ tapneumovirus (hMPV; 2\%; [20]). Damit sind zwar unverändert Rhinoviren für den Hauptanteil der Erkältungskrankheiten ursächlich, aber auch neuere Viren wie hMPV, Bocavirus oder neue Coronaviren spielen zunehmend eine Rolle.

Während in der nördlichen Hemisphäre RSV und Influenza von Januar bis März dominieren, können Parainfluenzaviren auch im Herbst und Frühwinter vorherrschen. Rhinoviren werden ganzjährig nachgewiesen, mit Gipfeln im Herbst, aber auch kleinen Ausbrüchen im Frühjahr (• Tab. 5). Bei der Übertragung sind Aerosole sowie kontaminierte Hände relevant.

Pathogenetisch werden Erkältungen durch eine komplexe Interaktion replizierender Viren und entzündlicher Reaktionen verursacht. Rhinoviren beispielsweise besiedeln die vordere Nasenschleimhaut und das Auge, wandern durch den Ductus nasolacrimalis zu den Adenoiden, dringen in die Epithelzellen ein und vermehren sich dort binnen 8-10 h nach der Ansteckung. Die Erkältungssymptome resultieren v. a. aus Vasodilatation (sog. Fließschnupfen) und vermehrter Gefäßdurchlässigkeit, aber auch aus einem Befall der Sinus und tieferen Atemwege. Von praktischer Relevanz ist, dass nur 75\% der Infizierten klinische Erkältungssymptome entwickeln, aber infektiös sind; dies unterstreicht die Wichtigkeit konsequenter Hygiene zur Erkältungsjahreszeit [9].

\section{Klinik}

Der klinische Verlauf variiert zwar mit dem ursächlichen Virus, allgemein aber nehmen die Beschwerden über 2 bis $3 \mathrm{Ta}$ ge zu, um danach bis zum 7. Erkrankungstag abzuklingen. Junge Kinder können bis zu 2 Wochen krank sein, eine Influenza kann in allen Altersgruppen 3 bis 4 Wochen dauern.

Eine Pharyngitis ist oft das erste Symptom, gefolgt von verstopfter Nase, Schnupfen und Husten. Während die Halsschmerzen bei viraler Genese meist rasch wieder verschwinden, kann die anfangs wässrige Rhinorrhö zunehmend purulent werden, was in der Regel kein Ausdruck einer behandlungsbedürftigen bakteriellen Infektion ist. Moderates Fieber begleitet häufig den Krankheitsverlauf; hohes Fieber, Lymphknotenschwellungen, starke Kopfschmerzen, produktiver Husten und mehr als 2 Wochen andauernde Symptome sollten an Komplikationen denken lassen.

Während bei älteren Kindern banale Erkältungen meist typische klinische Bilder (Rhinitis, Pharyngitis, Tracheobronchitis) bieten und manchmal allergische Erkrankungen der oberen Atemwege Mischbilder generieren können, kann bei jungen Kindern die Abgrenzung zu 
invasiven bakteriellen Infektionen schwerer fallen.

\section{Komplikationen}

Obwohl banale Erkältungen meist selbstlimitierende kurze Verläufe nehmen, können begleitende oder aufpfropfende bakterielle Infektionen auftreten. Bei Kindern steht dabei mit rund 20\% die akute Otitis media, die als Ausdruck einer Tubenfunktionsstörung 3 bis 4 Tage nach Erkältungsbeginn auftritt, im Vordergrund [14]. Andere nennenswerte Komplikationen sind bakterielle Sinusitiden (bei bis zu 2\% der Erkältungen), Pneumonien (oft als viral-bakterielle Mischinfektion) und Asthmaanfälle.

\section{Therapie}

In Anbetracht der komplexen viralen Ätiologie sind Behandlungsansätze im Wesentlichen symptomatisch. Antibiotika sind bei banalen Erkältungen meist entbehrlich und können keine Komplikationen verhindern, tragen aber zu bakteriellen Resistenzen bei.

\section{》) Selbstmedikationsprä- parate haben ein erhebliches Gefährdungspotenzial}

Alljährlich zur Winterzeit erhalten rund $10 \%$ der kindlichen Normalbevölkerung Selbstmedikationspräparate, die häufig Antipyretika, Antitussiva, Antikongestiva, Expektoranzien oder Antihistaminika enthalten. Für die meisten dieser Präparate gab es nie Dosisfindungsstudien, sondern die Dosierungsempfehlungen wurden gewichtsadaptiert von Erwachsenendosierungen abgeleitet. Solche Substanzen haben ihrerseits ein erhebliches Gefährdungspotenzial durch Überdosierungen, Neben- und Wechselwirkungen und sind der häufigste Vorstellungsgrund für Medikamentenunverträglichkeiten bei Kindern überhaupt. In den USA wird deshalb von Erkältungsselbstmedikation bei unter 6-Jährigen abgeraten [6].

Eltern sollten nicht nur über die potenziellen Risiken von Erkältungsmitteln bei Kindern aufgeklärt werden, sondern auch bezüglich der strittigen Wirksamkeits- nachweise. Zu Wirksamkeit von Erkältungsselbstmedikationen gibt es mittlerweile Cochrane-Analysen nach den Kriterien der evidenzbasierten Medizin. In Metaanalysen konnte keine Wirksamkeit von Selbstmedikationspräparaten bei ambulant behandelten Kindern mit viralen Erkältungsepisoden nachgewiesen werden [25, 27]. Primäre Endpunkte waren Dauer und Schweregrad des Hustens, Sputumproduktion, nasale Verstopfung und Einschätzung des Kinderarztes. Bei Erkältungen hatten Dekongestiva und Antihistaminika bei insbesondere jungen Kindern im Plazebovergleich keine nachgewiesene Wirksamkeit [3, 12]. Auch für Hustenblocker ist die Datenlage dünn; die American Academy of Pediatrics rät von Kodein oder Dextromethorphan enthaltenden Hustenmitteln ab.

In einer aktuellen nordamerikanischen Übersichtsarbeit zu therapeutischen Ansätzen bei erkältungskranken Kindern konnte keine Wirksamkeit für inhalative oder orale Steroide sowie Echinaceapräparate belegt werden [19]. Therapeutisch wirksam waren Vaporub ${ }^{\circledR}$ (ätherisches Kombinationspräparat aus Kamille, Eukalyptus und Menthol), Zinksulfat, Buchweizenhonig und Pelargonienwurzelextrakte (Umckaloabo $\left.{ }^{\circledR}, \mathrm{Kaloba}^{\circ}\right)$. Andere Therapieoptionen sind Honig, Kochsalzlösung (sog. Meerwasserspülung), Vitamin C, Echinacea und Zink [23]. In pädiatrischen Studien war Honig bei der Verminderung von Husten etablierten Hustenblockern (Dextromethorphan, Diphenhydramin) überlegen $[18,22]$ und stellt eine sinnvolle Behandlungsoption dar, sollte allerdings wegen der Gefahr des Säuglingsbotulismus erst jenseits des ersten Lebensjahres Einsatz finden. Kochsalzspülungen können muko- und sekretolytisch wirken, und Gurgeln mit warmer Kochsalzlösung hilft auch bei Halsschmerzen [24]. Insbesondere bei jungen Kindern kann auch physiologische Kochsalzlösung oder Muttermilch in die Nase eingeträufelt werden (Evidenzgrad C). Vitamin $\mathrm{C}$ zeigte in einer aktuellen Cochrane-Analyse bei Kindern kein therapeutisches Potenzial, spielt aber bei der Vorbeugung eine gewisse Rolle [10]. Eine gewisse prophylaktische Wirkung ließ sich für Zinksulfat (antiviral), Nasenspülung mit physiologischer Kochsalzlösung so- wie das Kombinationspräparat Chizukit (Echinacea, Vitamin C und Propolis) zeigen [5]. Bei 2- bis 10-Jährigen ließ sich während der Erkältungssaison mit prophylaktisch täglich $5 \mathrm{mg}$ Zinksulfat eine Verminderung der Häufigkeit und Schwere von Erkältungserkrankungen erreichen [16].

\section{Fazit für die Praxis}

- Infektionserkrankungen der oberen Atemwege zählen zu den häufigsten Gründen für eine kinderärztliche Konsultation und sollten eine topographisch-symptomorientierte Herangehensweise erfahren.

- Erkältungen sind in der Regel selbstlimitierende Erkrankungen, nur manchmal treten bakterielle Komplikationen (meist akute Otitis media) auf.

- Virusinfektionen stehen im Vordergrund, über seltene - aber schwer verlaufende - bakterielle Krankheitsbilder müssen sich die behandelnden Ärzte bewusst sein.

- Bei der Therapie klassischer Erkältungskrankheiten ist eine medikamentöse Polypragmasie zu vermeiden. Antibiotika werden oft auf Wunsch der Eltern eingesetzt; hier sollte auf mangelnde Sinnhaftigkeit und potenzielle Risiken hingewiesen werden. Antibiotika sind in Abwesenheit von Grunderkrankungen meistens entbehrlich, ihr Einsatz sollte kalkuliert erfolgen.

- Selbstmedikationspräparate für Erkältungen sind $v$. a. bei Kindern von fraglichem Nutzen und bergen Risiken.

- Mit der Verfügbarkeit von Schutzimpfungsprogrammen konnten Diphtherie und Haemophilus-influenzae-TypB-Erkrankungen sowie Pneumokokkeninfektionen erheblich zurückgedrängt werden, die neue nasale mukosale Kindergrippeimpfung verheißt zusätzliches Präventionspotenzial. 


\section{Korrespondenzadresse}

\section{Prof. Dr. M.A. Rose}

Klinik für Kinder- und Jugendmedizin, Sana-Klinikum Offenbach Starkenburgring 66, 63069 Offenbach Markus.Rose@klinikum-offenbach.de

\section{Einhaltung ethischer Richtlinien}

Interessenkonflikt. M.A. Rose hat Unterstützung für wissenschaftliche Projekte und Vortragshonorare der Firmen Abbott/Abbvie, AstraZeneca, GSK, Infectopharm, Novartis Vaccine, SPMSD, Symbiopharm, Wyeth/Pfitzer erhalten. Dies hat keine Auswirkungen auf die im Beitrag dargestellten Inhalte.

Alle im vorliegenden Manuskript beschriebenen Untersuchungen am Menschen wurden mit Zustimmung der zuständigen Ethik-Kommission, im Einklang mit nationalem Recht sowie gemäß der Deklaration von Helsinki von 1975 (in der aktuellen, überarbeiteten Fassung) durchgeführt. Von allen beteiligten Patienten liegt eine Einverständniserklärung vor. Alle nationalen Richtlinien zur Haltung und zum Umgang mit Labortieren wurden eingehalten und die notwendigen $\mathrm{Zu}$ stimmungen der zuständigen Behörden liegen vor.

\section{Literatur}

1. American Academy of Family Physicians (2004) The strength-of-recommendation taxonomy (SORT). AAFP, Leawood, KS, http:/www.aafp. org/afp/2004/0201/p548.html. Zugegriffen: 22.01.2014

2. Bjornson C, Russell K, Vandermeer B et al (2013) Nebulized epinephrine for croup in children. Cochrane Database Syst Rev 10:CD006619. DOI 10.1002/14651858.CD006619.pub3

3. Clemens CJ, Taylor JA, Almquist JR et al (1997) Is an antihistamine-decongestant combination effective in temporarily relieving symptoms of the common cold in preschool children? J Pediatr 130:463466

4. Deutsche Gesellschaft für pädiatrische Infektiologie (DPGI) (2013) DGPI Handbuch Infektionen bei Kindern und Jugendlichen, 6 . Aufl. Thieme, Stuttgart New York, S 574, 606-610

5. Fashner J, Ericson K, Werner S (2012) Treatment of the common cold in children and adults. Am Fam Physician 86:153-159

6. Food and Drug Administration (2013) FDA statement following CHPA's announcement on nonprescription over-the-counter cough and cold medicines in children. FDA, Silver Spring. http://www. fda.gov/newsEvents/Newsroom/PressAnnouncements/2008/ucm116964.htm. Zugegriffen: 10.01.2013

7. Garbutt JM, Conlon B, Sterkel R et al (2013) The comparative effectiveness of prednisolone and dexamethasone for children with croup: a community-based randomized trial. Clin Pediatr (Phila) 52:1014-1021

8. Glezen P, Gaglani MJ, Kozinetz CA, Piedra PA (2010) Direct and indirect effectiveness of influenza vaccination delivered to children at school preceding an epidemic caused by 3 new influenza virus variants. J Infect Dis 202:1626-1633

9. Heikkinen T, Jarvinen A (2003) The common cold. Lancet 361:51-59
10. Hemilä H, Chalker E, Douglas B (2007) Vitamin C for preventing and treating the common cold. Cochrane Database Syst Rev 3:CD000980

11. Hopkins A, Lahiri T, Salerno R et al (2006) Changing epidemiology of life-threatening upper airway infections: the re-emergence of bacterial tracheitis. Pediatrics 118:1418-1421

12. Hutton N, Wilson MH, Mellits ED et al (1991) Effectiveness of an antihistamine-decongestant combination for young children with the common cold: a randomized, controlled clinical trial. J Pediatr 118:125-130

13. Kairys SW, Olmstead EM, O'Connor GT (1989) Steroid treatment of laryngotracheitis: a meta-analysis of the evidence from randomized trials. Pediatrics 83:683-693

14. Kesson AM (2007) Respiratory virus infections. Paediatr Respir Rev 8:240-248

15. Kneen R, Nguyen MD, Solomon T et al (2004) Clinical features und predictors of diphteritic cardiomyopathy in Vietnamese children. Clin Infect Dis 39:1591-1598

16. Kurugöl A, Akilli M, Bayram N, Koturoglu G (2006) The prophylactic and therapeutic effectiveness of zinc sulphate on common cold in children. Acta Paediatr 95:1175-1181

17. Lemierre $A$ (1936) On certain septicaemias due to anaerobic organisms. Lancet 1:701-703

18. Paul IM, Beiler J, McMonagle A et al (2007) Effect of honey, dextromethorphan, and no treatment on nocturnal cough and sleep quality for coughing children and their parents. Arch Pediatr Adolesc Med 161:1140-1146

19. Robohm C, Ruff C (2012) Diagnosis and treatment of the common cold in pediatric patients. JAAPA 25:43-47

20. Ruohola A, Waris M, Allander T et al (2009) Viral etiology of common cold in children, Finland. Emerg Infect Dis 15:344-345

21. Salomone FN, Bobbitt DB, Myer CM et al (2004) Bacterial tracheitis re-examined: is there a less severe manifestation? Otolaryngol Head Neck Surg 131:871-876

22. Shadkam NM, Mozaffari-Khosravi $H$, Mozayan MR (2010) A comparison of the effect of honey, dextromethorphan, and diphenhydramine on nightly cough and sleep quality in children and their parents. J Altern Complement Med 16:787-793

23. Singh M, Das RR (2011) Zinc for the common cold. Cochrane Database Syst Rev 2:CD001364

24. Slapak I, Skoupá J, Strnad P, Horník P (2008) Efficacy of isotonic nasal wash (seawater) in the treatment and prevention of rhinitis in children. Arch Otolaryngol Head Neck Surg 134:67-74

25. Smith SM, Schroeder K, Fahey T (2008) Over the counter medications for acute cough in children and adults in ambulatory settings. Cochrane Database Syst Rev 1:CD001831

26. Stevens D, Ma Y, Salmi DB et al (2007) Impact of antibiotics on expression of virulence-associated exotoxin genes in methicillin-sensitive and methicillin-resistant Staphylococcus aureus. J Infect Dis 195:202-211

27. Sutter $\mathrm{Al}$, Lemiengre $\mathrm{M}$, Campbell $\mathrm{H}$, Mackinnon HF (2003) Antihistamines for the common cold. Cochrane Database Syst Rev 3:CD001267

28. Wierzbicka M, Jackowska J, Bartochowska A et al (2011) Effectiveness of cidofovir intralesional treatment in recurrent respiratory papillomatosis. Eur Arch Otorhinolaryngol 268:1305-1311

29. Zhang L, Sanguebsche LS (2005) The safety of nebulisation with 3 to $5 \mathrm{ml}$ of adrenaline (1:1000) in children: an evidence-based review. J Pediatr (Rio J) 81:193-197
30. Zooron R, Sidani M, Murray J (2011) Croup: an overview. Am Fam Physician 83:1067-1073

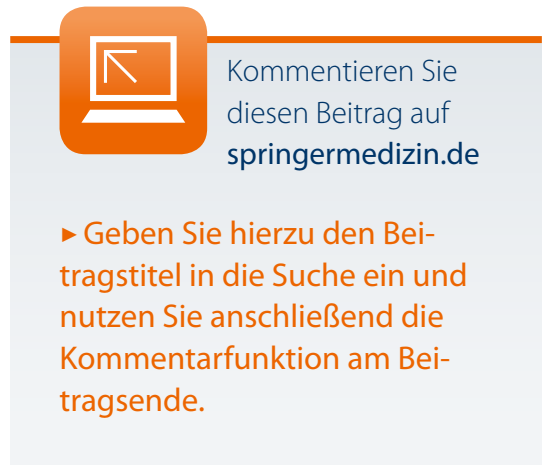

